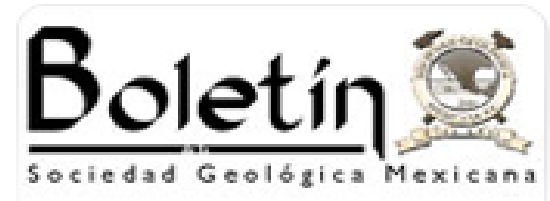

Boletín de la Sociedad Geológica Mexicana

ISSN: 1405-3322

sgm_editorial@geociencias.unam.mx

Sociedad Geológica Mexicana, A.C.

México

Bravo-Cuevas, Víctor M.; Ortiz-Caballero, Elizabeth; Cabral-Perdomo, Miguel Ángel Gliptodontes (Xenarthra, Glyptodontidae) del Pleistoceno Tardío (Rancholabreano) de Hidalgo, Centro de México

Boletín de la Sociedad Geológica Mexicana, vol. 61, núm. 2, 2009, pp. 267-276

Sociedad Geológica Mexicana, A.C.

Distrito Federal, México

Disponible en: http://www.redalyc.org/articulo.oa?id=94316034016

Cómo citar el artículo

Número completo

- Más información del artículo

Página de la revista en redalyc.org

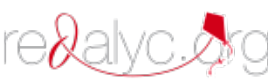

Sistema de Información Científica

Red de Revistas Científicas de América Latina, el Caribe, España y Portugal

Proyecto académico sin fines de lucro, desarrollado bajo la iniciativa de acceso abierto 
Boletín de la Sociedad Geológica Mexicana

VOLUMEN 61, NÚM. 2, 2009, P. 267-276

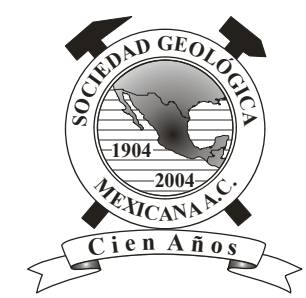

\title{
Gliptodontes (Xenarthra, Glyptodontidae) del Pleistoceno Tardío (Rancholabreano) de Hidalgo, Centro de México
}

\author{
Víctor M. Bravo-Cuevas ${ }^{1, *}$, Elizabeth Ortiz-Caballero², Miguel Ángel Cabral-Perdomo ${ }^{1}$ \\ ${ }^{1}$ Museo de Paleontología, Área Académica de Biología, Universidad Autónoma del Estado de Hidalgo. Ciudad Universitaria s/n, \\ Carretera Pachuca-Tulancingo km 4.5, CP 42184, Pachuca, Hidalgo, México. \\ ${ }^{2}$ Licenciatura en Biología, Universidad Autónoma del Estado de Hidalgo. Ciudad Universitaria s/n, Carretera Pachuca-Tulancingo km \\ 4.5, CP 42184, Pachuca, Hidalgo, México. \\ *vbravo@uaeh.edu.mx
}

\begin{abstract}
Resumen
Trabajo paleontológico realizado en la región centro-meridional de Hidalgo, permitió recuperar una muestra importante de restos pertenecientes a Glyptodontidae. El material se recuperó de una secuencia sedimentaria innominada que consiste en arcillas, limos y gravas de pobre a moderadamente consolidadas de origen fluviolacustre; los ejemplares están asociados a restos de bisonte (Bison), que sugiere una antigüedad asignable al Rancholabreano. La muestra incluye tres fragmentos de coraza y 10 osteodermos aislados. Los osteodermos son hexagonales con dos a cinco folículos pilosos, la figura central es poligonal, cóncava y ligeramente sobreelevada; alrededor de la figura central existen de ocho a nueve figuritas periféricas dispuestas de manera simétrica y configuración burdamente trapezoidal. El patrón de la roseta corresponde al observado en Glyptotherium floridanum y, por lo tanto, es que los ejemplares hidalguenses se asignan a esta especie. En México, registros de Glyptotherium provienen de Sonora, Chihuahua, Aguascalientes, Jalisco, Estado de México y Veracruz, en consecuencia, se reporta formalmente la presencia de este género para Hidalgo; asimismo, se anexa al registro previamente conocido y se amplia su rango de distribución en lo que ahora es parte del territorio hidalguense.

Palabras clave: Xenarthra, Glyptodontidae, Pleistoceno tardío, Centro de México.

Abstract

An important sample of Glyptodontidae remains was recovered from the south-central region of the State of Hidalgo. The fossil material was recovered from a fluviolacustrine unnamed sedimentary sequence that consists of poorly to moderate consolidated clays, silts, and gravels; the specimens are associated with remains of bison (Bison), which indicates a Rancholabrean Land Mammal Age. The sample includes two carapace fragments and 10 isolate osteoderms. The osteoderms are typical hexagonal with two to five pilose follicles, the central figure is polygonal, concave and slightly raised; there are eight to nine peripheral figures, smaller in size, roughly trapezoidal in shape, and symmetrically arranged around the center. All of these features are comparable to the rosette pattern of Glyptotherium floridanum, thus the specimens from Hidalgo are assigned to this species. In Mexico, the genus Glyptotherium is known from Sonora, Chihuahua, Aguascalientes, Jalisco, Estado de Mexico, and Veracruz. The presence of this taxon in Hidalgo is formally reported and is added to previously known record of North American glyptodonts, extending its distribution range to what is now part of central Mexico.
\end{abstract}

Keywords: Xenarthra, Glyptodontidae, late Pleistocene, Central Mexico. 


\section{Introducción}

El orden Xenarthra es un grupo de mamíferos de origen sudamericano, cuyos primeros registros provienen del Terciario temprano (Simpson, 1980). La mayor parte de su historia evolutiva ocurrió en dicho subcontinente; sin embargo, a partir del Mioceno tardío los xenartros comenzaron a invadir América del Norte y, eventualmente, fueron un componente importante de las faunas pleistocénicas norteamericanas (Kurtén y Anderson, 1980; Simpson, 1980; Webb y Rancy, 1996). Los xenartros integran un grupo monofilético que incluye a los subordenes Cingulata (armadillos, así como las formas emparentadas extintas gliptodontes y pampaterios), Vermilingua (osos hormigueros) y Phyllophaga (perezosos); éstos dos últimos se consideran tradicionalmente en el subgrupo Pilosa (Gaudin, 2003). En América del Norte se conocen restos fósiles de todos los grupos de xenartros, cuya evidencia en conjunto testifica la presencia de al menos 12 géneros con un alcance geocronológico del Mioceno tardío al Pleistoceno tardío. En México, se cuenta con alrededor del $80 \%$ de la diversidad genérica conocida, con un registro que incluye un oso hormiguero gigante (Myrmecophaga), un género de gliptodonte (Glyptotherium), dos géneros de pampaterios (Holmesina y Pampatherium) y seis géneros de perezosos terrestres (Pliometanastes, Megalonyx, Nothrotheriops, Eremotherium, "Glossotherium" y Paramylodon) (McDonald, 2002).

En particular, el estudio taxonómico detallado del registro mexicano perteneciente a Glyptodontidae (Cingulata) es aún escaso (Cuatáparo y Ramírez, 1875; Brown, 1912; Mead et al., 2007). Asimismo, se cuenta con algunos reportes que mencionan la presencia de estas formas en numerosas localidades del Pleistoceno de México (Hibbard, 1955; Silva-Bárcenas, 1969; Dalquest, 1961; Mooser y Dalquest, 1975; Montellano-Ballesteros, 1992; McDonald, 2002).

Los gliptodontes de América del Norte se han asignado al género Glyptotherium y actualmente se distinguen cinco especies, G. texanum (Blancano), G. arizonae (Irvingtoniano), G. floridanum (Rancholabreano), G. cylindricum (Rancholabreano) y G. mexicanum (Rancholabreano) (Gillette y Ray, 1981). En México, se cuenta con material perteneciente a los últimos tres taxones, procedente de localidades rancholabreanas en Sonora, Veracruz, Aguascalientes, Jalisco, Puebla y Estado de México (Castro-Azuara, 1997; McDonald, 2002; Mead et al., 2007); asimismo, se conocen ejemplares de identidad específica incierta asignados a Glyptotherium sp. del Pleistoceno tardío de Chihuahua, Estado de México y Distrito Federal (Silva-Bárcenas, 1969; McDonald, 2002). Con relación al registro, también existe evidencia de gliptodontes para el Blancano (3.9 - 3.1 Ma) de Guanajuato en la región central del país (Carranza-Castañeda y Miller, 2004; Flynn et al., 2005).

La información disponible muestra que la distribución de los gliptodontes en México es dispersa en regiones del norte, oriente y centro del país, así como al sur de la planicie costera del golfo; asimismo, la mayor parte del registro conocido procede de depósitos del Pleistoceno tardío. En esta línea de investigación, se caracterizó una colección de osteodermos (articulados y aislados) pertenecientes a Glyptotherium del Pleistoceno tardío del estado de Hidalgo, México. El propósito de este trabajo es presentar los resultados del estudio, incluyendo una comparación de la muestra hidalguense con ejemplares mexicanos de otras localidades, así como con material representativo de Estados Unidos.

\section{2. Área de estudio}

El material objeto de este estudio procede de tres localidades fosilíferas ubicadas en las áreas de San Agustín Tlaxiaca y Pachuca-Tulancingo en la porción centromeridional del estado de Hidalgo (Figura 1). En esta región del territorio hidalguense afloran rocas volcánicas y volcanosedimentarias del Terciario y Cuaternario (INEGI, 1992). En particular, se reconocen depósitos sedimentarios innominados del Cuaternario que forman abanicos aluviales y rellenos de valle, que consisten en arcillas y limos pobremente consolidados con intercalaciones irregulares de gravas volcánicas compuestas por fenoclastos que varían en tamaño de guijarros a bloques y composición intermedia a máfica (Bravo-Cuevas, 2002). El material de gliptodontes objeto del presente estudio procede de sedimentos de esta naturaleza; asimismo, cabe señalar que también se han recuperado restos fósiles de reptiles, roedores, osos, lobos, perezosos terrestres, caballos, camélidos, berrendos, cérvidos, bisontes, mamuts y mastodontes (Castillo-Cerón et al., 1996; Bravo-Cuevas, 2001, 2002; Cabral-Perdomo, 2001). La presencia de ejemplares pertenecientes al género Bison, indica una edad equivalente al Rancholabreano en la cronología de mamíferos (Bell et al., 2004).

\section{1. Área San Agustín Tlaxiaca}

Esta área se encuentra en la región centro-meridional del Estado de Hidalgo, entre los $20^{\circ} 04^{\prime}$ - $20^{\circ} 14^{\prime}$ Lat. N y los 9849' - 9900' Long. O. Las localidades fosilíferas de esta parte del territorio hidalguense incluyen a "Las Cajas" (HGO-46) en los 2007'31.7" Lat. N - 98 58'07.1" Long. $\mathrm{O}$ a unos $2140 \mathrm{msnm}$, así como "El Barrio" (HGO-47) en los 2007'41" Lat. N - 98 56'02.7”' Long. O a 2184 msnm (Figura 2A).

\section{2. Área Pachuca-Tulancingo}

El área Pachuca-Tulancingo forma parte del límite norte de la Cuenca de México, en el centro-sureste de Hidalgo, entre los $20^{\circ} 00^{\prime} \mathrm{y} \operatorname{los} 20^{\circ} 15^{\prime}$ Lat. N y los $98^{\circ} 19^{\prime}$ y $98^{\circ} 50^{\prime}$ Long. O. La localidad de la cual procede el material fósil se conoce como "Barranca del Berrendo" (HGO-28) en los $20^{\circ} 01^{\prime 2} 20.7^{\prime \prime}$ Lat. N - 98³7'37.9” Long. O a 2458 msnm 


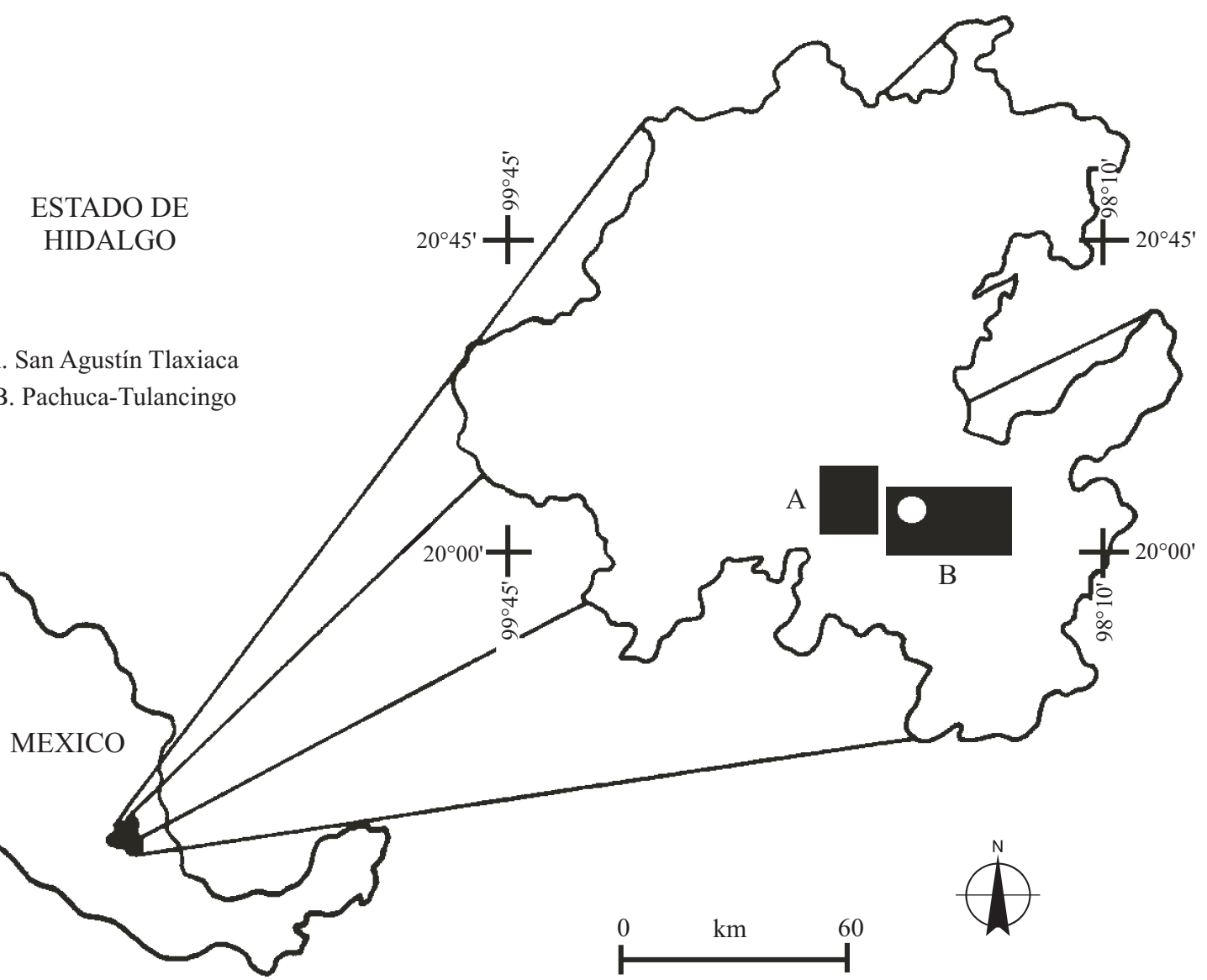

Figura 1. Mapa índice de las áreas de estudio en la región centro-meridional de territorio hidalguense: A. San Agustín Tlaxiaca y B. Pachuca-Tulancingo. También se denota con un punto blanco la capital del estado, Pachuca.
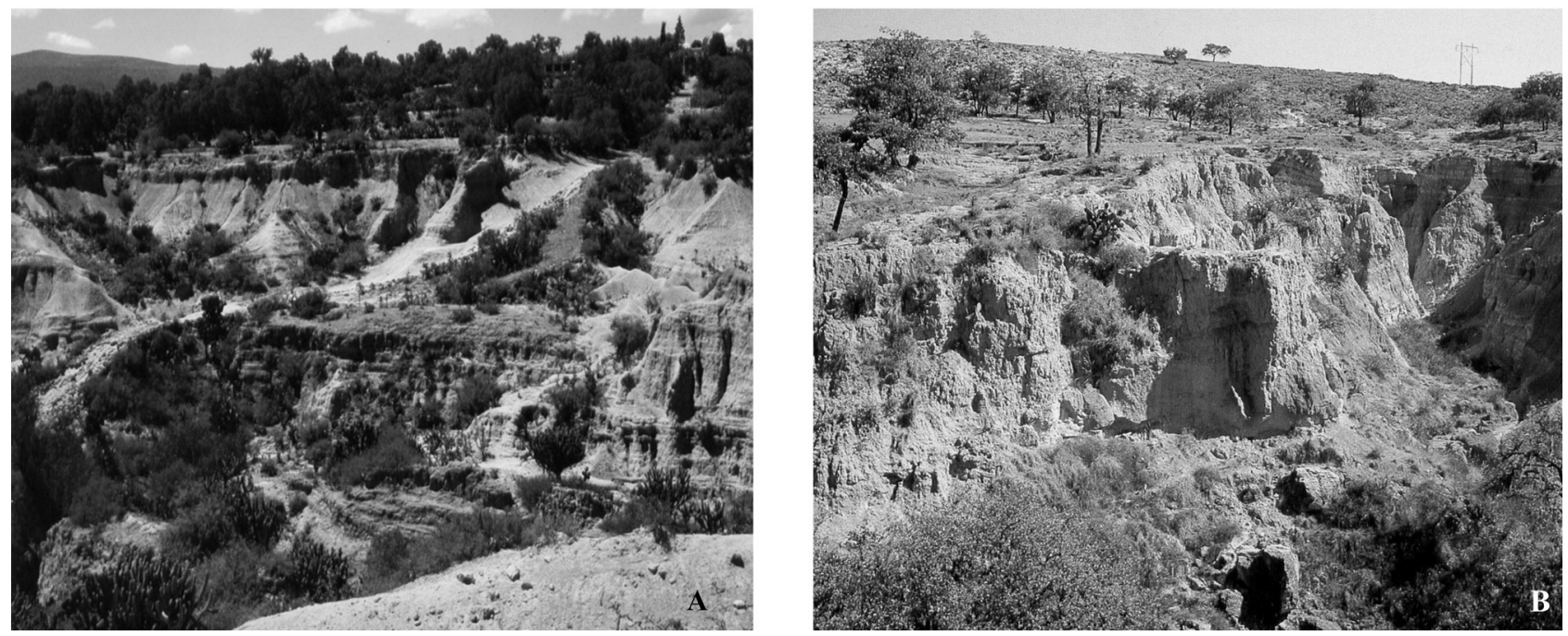

Figura 2. Vista panorámica de las localidades fosilíferas de la cuales procede el material de Glyptodontidae considerado en el presente estudio. A. Panorámica de la localidad "El Barrio" (HGO-47) en el área San Agustín Tlaxiaca y B. Panorámica de la localidad "Barranca del Berrendo" (HGO-28) en el área Pachuca-Tulancingo. 
(Figura 2B).

\section{Materiales y Métodos}

En este estudio se describe todo el material perteneciente a Glyptodontidae, procedente de localidades fosilíferas ubicadas en las áreas San Agustín Tlaxiaca y PachucaTulancingo, región centro-meridional del territorio hidalguense. El registro está albergado en la Colección de Macromamíferos del Museo de Paleontología de la Universidad Autónoma del Estado de Hidalgo (UAHMP).

La muestra incluye tres conjuntos de osteodermos articulados y diez aislados. Para cada uno de los ejemplares se evaluó un conjunto de rasgos configuracionales y métricos, ampliamente utilizados en la taxonomía y sistemática de xenartros (Gillette y Ray, 1981; Gaudin, 2003; Hill, 2005, 2006). La terminología y nomenclatura empleadas para la descripción de los osteodermos corresponde a la de Gillette y Ray (1981) y Hill (2006).

La asignación taxonómica se baso en la comparación de la muestra disponible con material de gliptodontes albergado en las colecciones del Museo Americano de Historia Natural en Nueva York (AMNH, American Museum of Natural History), el Museo Memorial de Texas en Austin (TMM, Texas Memorial Museum) y el Museo de Historia Natural de Florida (UF/FGS, Vertebrate Paleontology Collection, Florida Geological Survey, Museum of Natural History at University of Florida); asimismo, se revisaron descripciones originales e ilustraciones de ejemplares representativos previamente publicadas en Kurtén y Anderson (1980), Gillette y Ray (1981), Webb (2001), Hill (2006) y Mead et al. (2007).

\section{Paleontología Sistemática}

Orden Xenarthra Cope, 1889

Suborden Cingulata Illiger, 1811

Familia Glyptodontidae Gray, 1869

Género Glyptotherium Osborn, 1903

Glyptotherium floridanum Simpson, 1929

Tabla 1, Figuras 3-5

\subsection{Material referido}

Área San Agustín Tlaxiaca.- Localidad HGO-46: UAHMP - 428, osteodermo aislado del borde caudal; UAHMP-429 y UAHMP-431, dos osteodermos aislados de la región dorsal. Localidad HGO-47: UAHMP-426, conjunto de 20 osteodermos articulados de la región lateral; UAHMP- 427, conjunto de 13 osteodermos articulados de la región dorsal; UAHMP-430 y UAHMP-960, osteodermos aislados de la zona lateral; UAHMP - 967, osteodermo proximal de anillo caudal. Área Pachuca - Tulancingo.Localidad HGO-28: UAHMP - 358, conjunto de cinco osteodermos articulados de la región dorsal; UAHMP 919A, osteodermo distal de anillo caudal; UAHMP-919B, osteodermo del borde lateral; UAHMP-919D y UAHMP919E, osteodermos de la región lateral.

\subsection{Alcance geocronológico y distribución geográfica}

En América del Norte, el registro de Glyptotherium floridanum procede del Rancholabreano (Pleistoceno tardío, 0.3 - 0.011 Ma) de Carolina del Sur, Texas y Florida en los Estados Unidos (Roth y Laerm, 1980; Webb, 2001), así como de Veracruz, Aguascalientes (Dalquest, 1961; Mooser y Dalquest, 1975; McDonald, 2002) e Hidalgo (este estudio) en la República Mexicana.

\subsection{Descripción}

El material disponible incluye osteodermos articulados $\mathrm{y}$ aislados que forman parte de las regiones dorsal, lateral y marginal de la coraza, así como de la coraza caudal. Con base en esto, los ejemplares se describen según la zona a la que pertenecen y atendiendo a sus características distintivas.

\subsubsection{Osteodermos de la región dorsal (Figura 3)}

Los osteodermos son hexagonales, con un diámetro promedio antero-posterior de $42.5 \mathrm{~mm}$ y transverso de 36.17 $\mathrm{mm}$. El espesor tiene un rango de 14 - $17 \mathrm{~mm}$ (Tabla 1); sin embargo, en UAHMP-358 los elementos que integran el conjunto de cinco osteodermos articulados tienen mayor espesor ( $24.7 \mathrm{~mm}$ en promedio). La figura central esta ligeramente sobreelevada, es de contorno poligonal, ocupa del $38 \%$ al $40 \%$ del diámetro antero-posterior y su superficie es cóncava; en los ejemplares UAHMP-429 y UAHMP-431 la figura central es ligeramente cóncava. Alrededor de la figura central se observan de ocho a nueve figuritas periféricas de forma poligonal y menor tamaño; asimismo, se reconocen de tres a cinco folículos pilosos. La superficie ventral de los osteodermos es cóncava a ligeramente cóncava y, frecuentemente está perforada por uno a tres forámenes neurovasculares bien desarrollados.

\subsubsection{Osteodermos de la región lateral (Figura 4)}

El patrón de estos osteodermos es semejante al de aquellos pertenecientes a la región dorsal de la coraza. En particular, los osteodermos tienen un diámetro promedio antero-posterior de $43.5 \mathrm{~mm}$ y transverso de $34.4 \mathrm{~mm}$, mientras que el espesor es de alrededor de $15 \mathrm{~mm}$. La figura central ocupa ca. 35\% del diámetro antero-posterior del escudo (Tabla 1). Estos elementos se distinguen por presentar una superficie ventral plana, ocasionalmente perforada por uno o dos forámenes neurovasculares.

\subsubsection{Osteodermos de la región marginal}

La muestra disponible incluye dos osteodermos aislados de esta región de la coraza dorsal. El ejemplar UAHMP919B es un osteodermo del margen lateral de la coraza, 
Tabla 1. Medidas (mm) de los osteodermos de Glyptotherium floridanum del Pleistoceno tardío (Rancholabreano) de la región centro-meridional de Hidalgo, Centro de México.

\begin{tabular}{lcccccc}
\hline $\begin{array}{c}\text { Número de } \\
\text { catálogo }\end{array}$ & $\begin{array}{c}\text { Región de la } \\
\text { coraza }\end{array}$ & $\begin{array}{c}\text { Diámetro } \\
\text { antero-posterior }\end{array}$ & $\begin{array}{c}\text { Diámetro } \\
\text { transverso }\end{array}$ & $\begin{array}{c}\text { Diámetro de la } \\
\text { figura central }\end{array}$ & $\begin{array}{c}\text { Espesor de los } \\
\text { osteodermos }\end{array}$ & $\begin{array}{c}\text { Porcentaje que } \\
\text { ocupa la figura } \\
\text { central }\end{array}$ \\
\hline UAHMP-358 & Dorsal & $44.18-47.86$ & $35.78-44.94$ & $15.64-19.65$ & $23.3-26.74$ & $34.23 \%-41.4 \%$ \\
UAHMP-426 & Lateral & $43.26-49.79$ & $28.14-35.28$ & $15.2-18.56$ & $13.0-14.73$ & $32.79 \%-42.9 \%$ \\
UAHMP-427 & Dorsal & $43.15-46.86$ & $31.71-34.6$ & $15.64-19.65$ & $15.2-16.85$ & $36.16 \%-40.68 \%$ \\
UAHMP-429 & Dorsal & 43.07 & 39.83 & 17.11 & 16.24 & $39.72 \%$ \\
UAHMP-430 & Lateral & 49.46 & 42.74 & 20.42 & 18.75 & $41.28 \%$ \\
UAHMP-431 & Dorsal & 37.41 & ----- & 14.95 & 14.37 & $39.96 \%$ \\
UAHMP-919B & Lateral & 40.9 & 30.78 & 25.83 & 14.99 & $63.15 \%$ \\
UAHMP-919D & Lateral & ----- & ----- & ----- & 17.16 & --15 \\
UAHMP-919E & Lateral & 38.68 & 33.6 & 17.24 & 15.32 & $44.57 \%$ \\
UAHMP-960 & Lateral & 42.25 & 37.83 & 16.49 & 15.88 & $39.02 \%$ \\
\hline
\end{tabular}
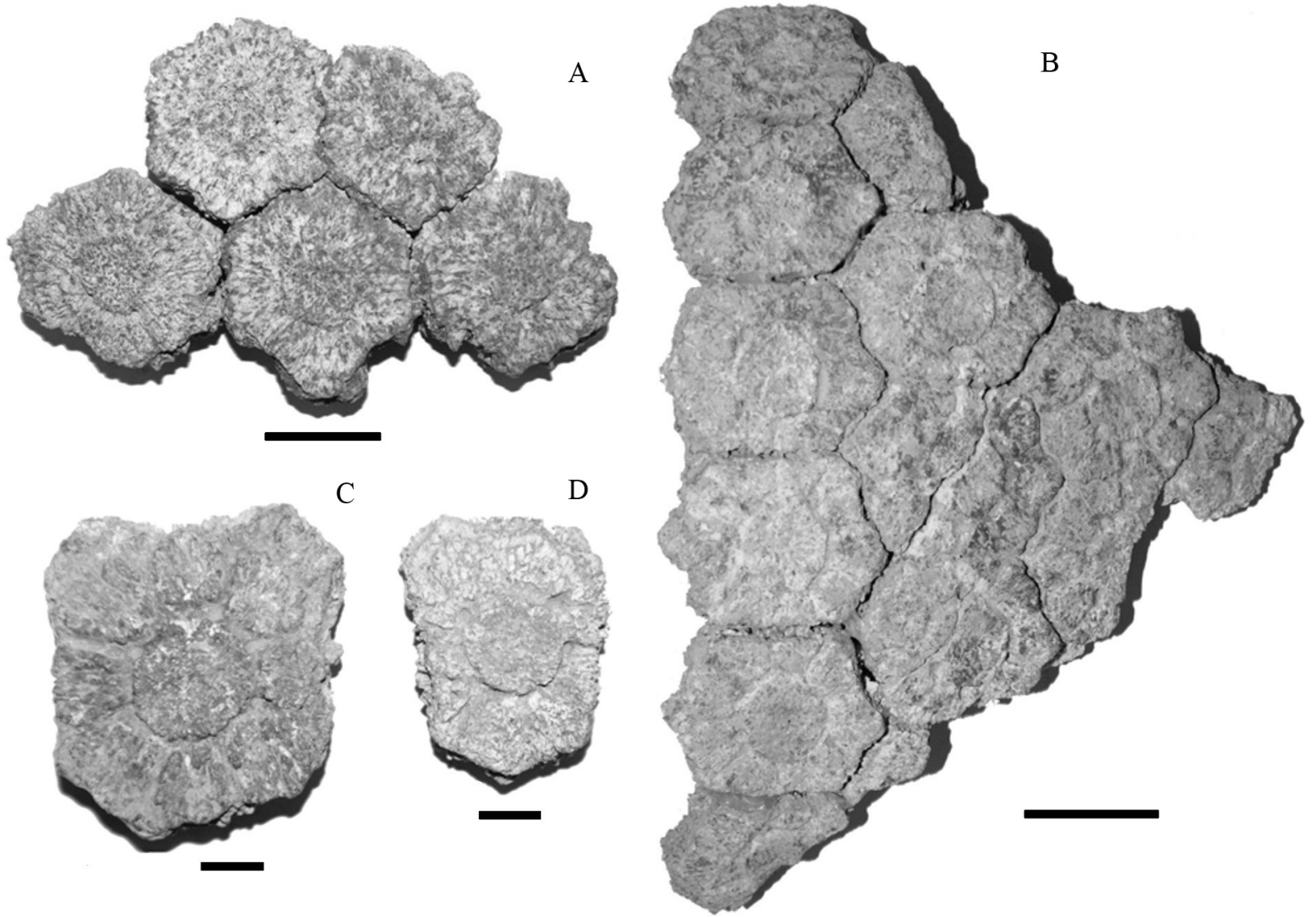

Figura 3. Osteodermos de la región dorsal de la coraza referidos a Glyptotherium floridanum del Pleistoceno tardío (Rancholabreano) de Hidalgo, Centro de México. A. Vista dorsal de UAHMP - 358, conjunto de cinco osteodermos articulados. B. Vista dorsal de UAHMP- 427, fragmento de coraza con 13 osteodermos articulados. C-D. Vista dorsal de UAHMP-429 y UAHMP-431 respectivamente. Escala = $3 \mathrm{~cm}($ A y B), Escala = $1 \mathrm{~cm}(\mathrm{C}$ y D).

tiene una configuración trapezoidal, así como diámetro antero-posterior de $40.9 \mathrm{~mm}$ y diámetro transverso de 30.78 $\mathrm{mm}$. El espesor de este elemento es de aproximadamente $25 \mathrm{~mm}$. La figura central es ovoidal y ocupa la mayor parte del osteodermo, mientras que las figuritas periféricas son prácticamente indistinguibles; asimismo, se observa un folículo piloso. La superficie ventral es ligeramente cóncava (Figura 5A).

El ejemplar UAHMP-428 corresponde a un osteodermo del borde caudal, tiene una configuración pentagonal, diámetro antero-posterior de $45.80 \mathrm{~mm}$ y diámetro transverso de $41.83 \mathrm{~mm}$, así como tiene un espesor de 

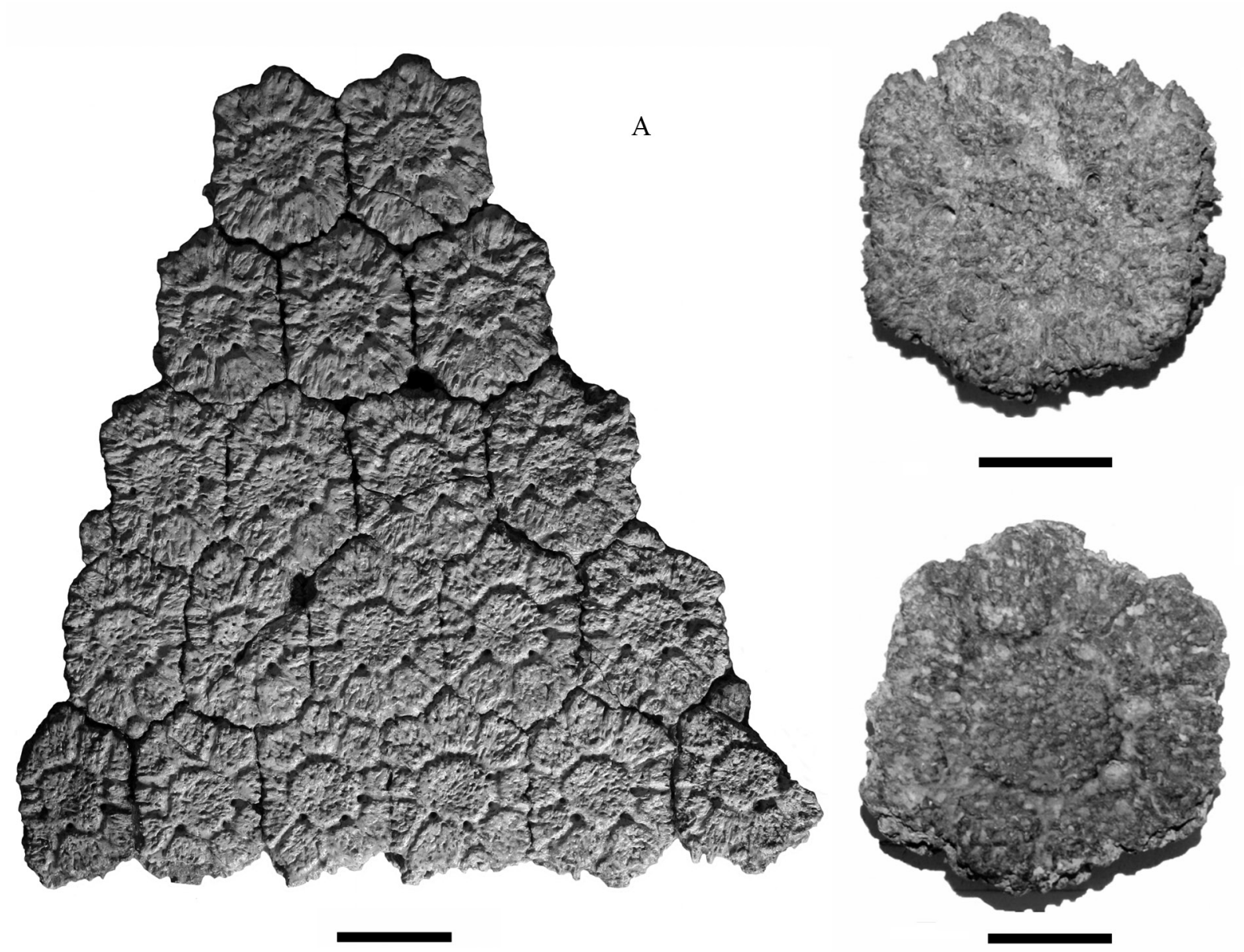

B

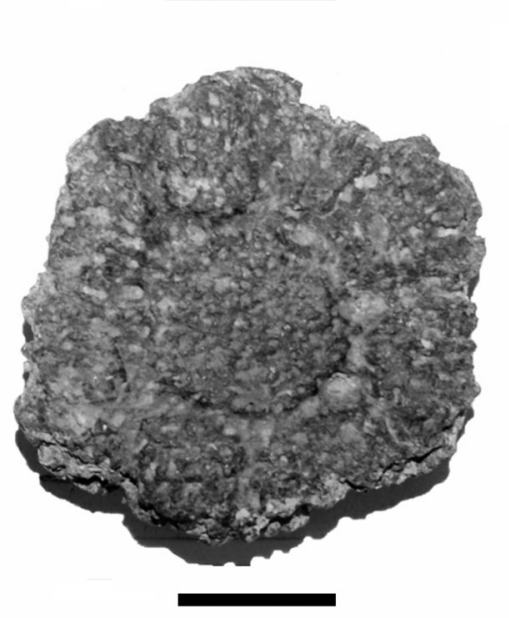

Figura 4. Osteodermos de la región lateral de la coraza referidos a Glyptotherium floridanum del Pleistoceno tardío (Rancholabreano) de Hidalgo, Centro de México. A. Vista dorsal de UAHMP-426, fragmento de coraza con 20 osteodermos articulados. B- C. Vista dorsal de UAHMP-960 y UAHMP-430 respectivamente, osteodermos aislados. Escala $=3 \mathrm{~cm}$.

aproximadamente $24 \mathrm{~mm}$ a nivel de la sutura interior. La superficie dorsal es rugosa y fuertemente convexa, mientras que la ventral es plana (Figura 5B).

\subsubsection{Coraza caudal}

La muestra estudiada incluye dos osteodermos aislados que forman parte de los anillos caudales. El ejemplar UAHMP-967 es poligonal con un diámetro anteroposterior de $34.17 \mathrm{~mm}$ y diámetro transverso de $30.21 \mathrm{~mm}$; asimismo, tiene un espesor de aproximadamente $11 \mathrm{~mm}$. La superficie dorsal es ligeramente convexa, ornamentada por múltiples estriaciones y fosas dispuestas de manera radial. La superficie ventral es plana y ligeramente oblicua con respecto al plano longitudinal, mientras que hacia la mitad posterior es fuertemente cóncava (Figura 5C).

El ejemplar UAHMP-919A tiene un diámetro anteroposterior de $51.47 \mathrm{~mm}$ y diámetro transverso de 43.89 $\mathrm{mm}$, así como un espesor de $23 \mathrm{~mm}$. La superficie dorsal es cónica y esta ornamentada por numerosas fosas. La superficie ventral es rugosa y ligeramente cóncava (Figura $5 \mathrm{D})$.

\section{Discusión}

La comparación del conjunto de osteodermos de gliptodontes de Hidalgo se llevo al cabo exclusivamente con las especies rancholabreanas Glyptotherium cylindricum, G. floridanum y G. mexicanum, dado que, el material correspondiente está asociado a ejemplares pertenecientes al género Bison, fósil estratigráfico del Rancholabreano en las edades de mamíferos (Bell et al., 2004). Con base en esto, se excluyeron a los taxones norteamericanos $G$. texanum y G. arizonae, los cuales se conocen del Blancano tardío al Irvingtoniano del Sur de la Gran Cuenca y Planicie Costera del Golfo (Kurtén y Anderson, 1980; Gillette y Ray, 1981).

Parte de la taxonomía de los gliptodontes se basa en el patrón de ornamentación de los osteodermos de la coraza dorsal, debido a que la forma, tamaño y arreglo de la figura central, así como de las figuritas periféricas, produce un patrón diagnóstico (Gillette y Ray, 1981; Webb, 2001; Hill, 

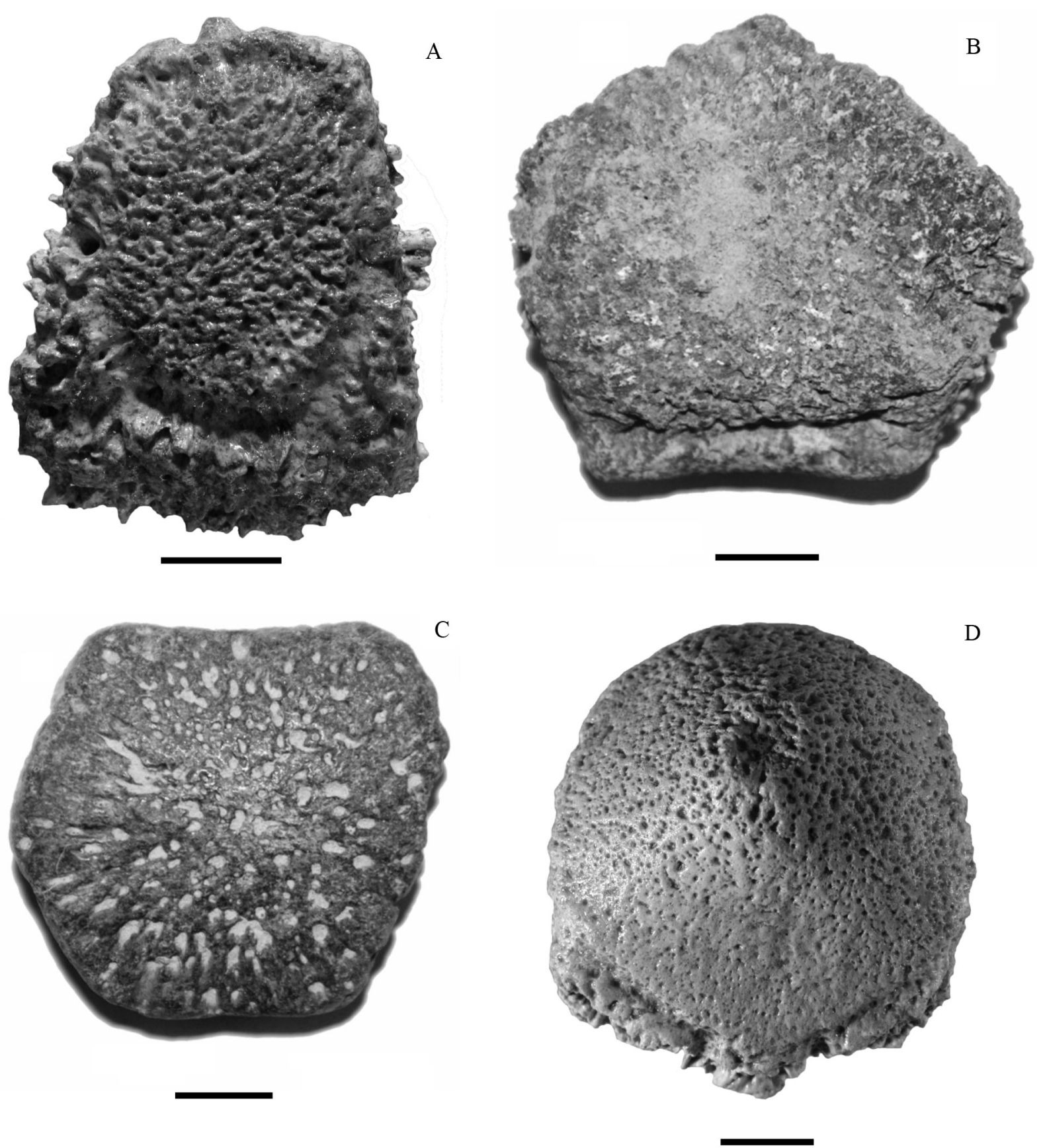

Figura 5. Osteodermos aislados (vista dorsal) de Glyptotherium floridanum del Pleistoceno tardío (Rancholabreano) de Hidalgo, Centro de México. A. UAHMP-919B, osteodermo del borde lateral de la coraza. B. UAHMP-428, osteodermo del margen caudal de la coraza. C. UAHMP-967, osteodermo proximal de anillo caudal. D. UAHMP-919A, osteodermo distal de anillo caudal. Escala $=1 \mathrm{~cm}$.

2006). Con relación a esto, el patrón de los osteodermos de los ejemplares hidalguenses es comparable al de Glyptotherium floridanum en la siguiente combinación de caracteres: la figura central está elevada y su superficie es cóncava, es ligeramente más grande que las periféricas y ocupa menos del $50 \%$ del diámetro total del osteodermo. Por su parte, G. cylindricum difiere en presentar figuras centrales con una superficie que varía de plana a ligeramente convexa, son más grandes que las periféricas y ocupan aproximadamente el $50 \%$ del diámetro total del osteodermo. Finalmente, G. mexicanum se distingue por tener figuras centrales más grandes que las periféricas y ocupan generalmente más del 50\% del diámetro del osteodermo (Gillette y Ray, 1981; Mead et al., 2007). 
La morfología de los osteodermos hidalguenses es estrechamente cercana a la de material referido a Glyptotherium floridanum, tal es el caso de AMNH 23457 (holotipo que incluye un fragmento de coraza de la región dorsal del borde caudal) y UF/FGS 6643 (un fragmento de coraza con 36 osteodermos articulados), procedentes de las localidades Seminole Field y Catalina Gardens Pinellas respectivamente, Rancholabreano de Florida ( $c f$. Gillette y Ray, 1981: figs. 88 y 90). En estos ejemplares, al igual que en la muestra considerada en este estudio, los osteodermos se caracterizan por presentar una figura central con una superficie cóncava ornamentada por numerosas fosas de tamaño pequeño, ligeramente elevada por encima del nivel de las periféricas y, relativamente más grande que las figuritas periféricas; asimismo, se reconoce un número semejante de figuras periféricas (ocho en promedio) y folículos pilosos (tres en promedio). Por otra parte, el tamaño promedio de los osteodermos que integran a AMNH 23457 y UF/FGS 6643 (ca. $45 \mathrm{~mm}$ de longitud antero-posterior), es comparable al que se reconoció para el material hidalguense ( $c a .43 \mathrm{~mm}$ de longitud antero-posterior).

La forma y tamaño de UAHMP-428 corresponden a las de los escudos del borde caudal de AMNH 23457. Un caso semejante ocurre cuando el osteodermo hidalguense se compara con AMNH 95728, un escudo aislado del borde caudal atribuido a un individuo macho de G. floridanum, procedente de Seminole Field, Rancholabreano de Florida (cf. Gillette y Ray, 1981: fig. 89); sin embargo, este último ejemplar es de mayor tamaño (aproximadamente un 30\% más grande).

La coraza caudal de los gliptodontes (así como la de los armadillos actuales) esta formada por ocho a diez anillos concéntricos, cada uno de ellos regularmente compuesto por dos filas de osteodermos. Con relación a esto, al comparar los ejemplares UAHMP-919 A y UAHMP-967 con una coraza caudal de Glyptotherium floridanum recuperada de la localidad Ingleside del Rancholabraenao de Texas (Gillette y Ray, 1981: fig. 94, TMM977-3), se observa que la configuración de los osteodermos hidalguenses es típica de aquellos que forman parte de los anillos caudales. En particular, la configuración poligonal, el arqueamiento y ornamentación de la superficie (numerosas fosas y estriaciones) que distinguen a UAHMP-967, sugieren que el ejemplar hidalguense pudiese corresponder a uno de los escudos de la fila distal del tercer anillo caudal o de los que forman las filas proximales del resto de los anillos; esta interpretación es consistente con el hecho de que los osteodermos del primer y segundo anillo son cuadrilaterales, aplanados (en sentido dorso-ventral) y superficie lisa, mientras que aquellos de la fila distal del anillo cuatro en adelante son cónicos. Por su parte, la configuración cónica de UAHMP-919A es típica de los osteodermos de la serie distal de los anillos caudales cuatro en adelante, aunado a esto, el tamaño del escudo hidalguense (diámetro transverso de $43.89 \mathrm{~mm}$ ) es comparable al que se observa en los osteodermos correspondientes a la coraza caudal de
TMM977-3 (diámetro transverso promedio de $41.6 \mathrm{~mm}$ ) (Gillette y Ray, 1981).

En resumen, el estudio comparado conduce a considerar que los ejemplares hidalguenses son estrechamente cercanos en tamaño y patrón del osteodermo que distinguen a Glyptotherium floridanum. Con base en esto, así como atendiendo a la edad sugerida de los sedimentos de los cuales se recuperó la muestra estudiada, así como al amplio valor taxonómico que tienen los osteodermos en la identificación de gliptodontes ( $c f$. Kurtén y Anderson, 1980; Gillette y Ray, 1981; Webb, 2001; Hill, 2006), es que el material de Hidalgo se asigna a dicha especie.

Los primeros reportes de gliptodontes en América del Norte se hicieron con base en material fósil mexicano (Cuatáparo y Ramírez, 1875); sin embargo, es escasa la información que se tiene de este grupo de xenartros para el territorio nacional. Como se mencionó en párrafos precedentes, para el Pleistoceno tardío (Rancholabreano) de México se conocen tres especies de gliptodontes (Glyptotherium mexicanum, G. cylindricum y $G$. floridanum). Con relación a esto, cabe señalar aquí que el status taxonómico de Glyptotherium mexicanum es incierto debido a que la descripción original es ambigua (Cuatáparo y Ramírez, 1875), aunado a esto, los ejemplares en los que se basó su asignación taxonómica están perdidos (Gillette y Ray, 1981). Por otra parte, con relación a Glyptotherium cylindricum y Glyptotherium floridanum, algunos autores consideran que son formas estrechamente cercanas en morfología y sugieren su probable sinonimia (Gillette y Ray, 1981; Mead et al., 2007). De lo anterior, se sigue que la posición taxonómica de los gliptodontes mexicanos es aún controversial y, por lo tanto, es necesario llevar al cabo estudios exhaustivos al respecto, que conduzcan a esclarecer la identidad específica de los taxones que hasta ahora se considera habitaron el territorio nacional durante la última parte del Pleistoceno. Aún con estas limitaciones y atendiendo a la taxonomía vigente propuesta para los gliptodontes de México, la morfología y tamaño de la muestra hidalguense son mucho más cercanas a las que se proponen para G. floridanum.

\section{Conclusiones}

El presente trabajo reporta formalmente y por primera vez la presencia de ejemplares fósiles pertenecientes a $G$. floridanum para el Rancholabreano del estado de Hidalgo. Aunado a esto, el material hidalguense aporta evidencia adicional sobre la distribución de esta especie en el centro de México, que había sido previamente considerada como circunscrita a la planicie costera del Golfo (Gillette y Ray, 1981; McDonald, 2002), complementando así el área de distribución en el territorio nacional y el subcontinente Norteamericano en conjunto.

Finalmente, el presente estudio es una contribución al conocimiento de los xenartros pleistocénicos de México, 
específicamente de los gliptodontes, que se anexa a la base factual disponible al respecto y servirá de comparación para el desarrollo de futuros trabajos que coadyuven al mejor entendimiento de una parte de la megafauna del Pleistoceno del país

\section{Agradecimientos}

Agradecemos en primera instancia a Greg McDonald y Alfredo Eduardo Zurita por la revisión critica realizada al manuscrito y cuyos comentarios enriquecieron sustancialmente la versión definitiva del presente trabajo. Expresamos nuestro agradecimiento a la Universidad Autónoma del Estado de Hidalgo por el apoyo económico otorgado para la realización de trabajo de campo en la región de San Agustín Tlaxiaca, durante el periodo 20002001, mediante el proyecto PSO-6MOD "Diferenciación geológica y bioestratigráfica de la Formación Tarango en el estado de Hidalgo, Centro de México", Instituto de Investigaciones en Ciencias de la Tierra, UAEH. Asimismo, agradecemos al C. Tomas Angeles Hernández, profesor de Inglés de la Universidad Autónoma del Estado de Hidalgo, por su participación en la prospección y rescate de una parte del material de gliptodontes que se describe en este estudio

\section{Referencias}

Bell, C.J., Lundelius Jr., E.L., Barnosky, A.D., Graham, R.W., Lindsay, E.H., Ruez Jr., D.R., Semken Jr., H.A., Webb, S.D., Zakrzewski, R.J., 2004, The Blancan, Irvingtonian, and Rancholabrean mammal ages, in Woodburne, M.O. (ed.), Late Cretaceous and Cenozoic mammals of North America: New York, Columbia University Press, 232-314.

Bravo-Cuevas, V.M., 2001, Pleistocene faunal assemblage from southcentral region of the state of Hidalgo, Central Mexico (abstract), in 61 th Annual Meeting Society of Vertebrate paleontology: Bozeman, Montana, Journal of Vertebrate Paleontology, Abstracts of papers, $21,35 \mathrm{~A}$.

Bravo-Cuevas, V.M., 2002, Diferenciación Geológica y bioestratigráfica de la formación Tarango para el estado de Hidalgo, Centro de México: Hidalgo, México, Universidad Autónoma del Estado de Hidalgo, Centro de Investigaciones en Ciencias de la Tierra, Reporte Técnico.

Bravo-Cuevas, V.M., Cabral-Perdomo, M.A., 2002, El primer registro del género Odocoileus para el estado de Hidalgo, Centro de México (resumen), en VIII Congreso Nacional de Paleontología, Guadalajara, Jalisco, Museo de Paleontología de Guadalajara, 35.

Brown, B., 1912, Brachyostracon, a new genus of glyptodont from Mexico: American Museum of Natural History Bulletin, 31, 167-177.

Cabral-Perdomo, M.A., 2001, Vertebrados pleistocénicos de la región Pachuca-Tulancingo, estado de Hidalgo: México, Universidad Nacional Autónoma de México, Facultad de Ciencias, tesis de Maestría.

Castillo-Cerón, J.M., Cabral-Perdomo, M.A., Carranza-Castañeda, O., 1996, Vertebrados fósiles del Estado de Hidalgo: Hidalgo, México, Universidad Autónoma del Estado de Hidalgo.

Carranza-Castañeda, O., Miller, W.E., 2004, Late Tertiary terrestrial mammals from central México and their relationship to South American inmigrants: Revista Brasileira de Paleontologia, 7, 249-261.

Castro-Azuara, H.E., 1997, Descripción de un gliptodonte (Xenarthra: Mammalia) del Pleistoceno de la Mixteca alta Poblana, México:
Puebla, México, Benemérita Universidad Autónoma de Puebla, Escuela de Biología, tesis de Licenciatura.

Cope, E.D., 1889, The Edentata of North America: American Naturalist, 23, 659-664.

Cuatáparo, J.N., Ramírez., S., 1875, Descripción de un mamífero fósil de especie desconocida perteneciente al género Glyptodon: Boletín Sociedad Mexicana de Geografía y Estadística, 2, 354-362.

Dalquest, W.W., 1961, Sylvilagus cunicularius in the Pleistocene of México: Journal of Mammalogy, 42, 408-409.

Flynn, J.J., Kowallis, B.J., Nuñez, C., Carranza Castañeda, O., Miller, W.E., Swisher III, C.C., Lindsay, E., 2005, Geochronology of HemphillianBlancan aged strata, Guanajuato, México, and Implications for timing of the Great American biotic interchange: Journal of Geology, 113, 287-307.

Gaudin, T.J., 2003, Phylogeny of the Xenarthra, in Fariña, R.A., Vizcaíno, S.F., Storch, G. (eds.), Morphological Studies in Fossil and Extant Xenarthra (Mammalia): Frankfurt am Main, Seckenbergiana Biologica, 27-40.

Gillette, D.D., Ray, C.E., 1981, Glyptodonts of North America: Smithsonian Contributions to Paleobiology, 40, 1-255.

Gray, J.E., 1869, Catalogue of carnivorous, pachydermatous, and dentate mammalia in the Brithish Museum: London, British Museum (Natural History).

Hibbard, C.W., 1955, Pleistocene vertebrates from the Upper Becerra (Becerra Superior) Formation, Valley of Tequixquiac, México, with notes on other Pleistocene forms: University of Michigan Museum of Paleontology, Contributions, 12, 47-96.

Hill, R.V., 2005, Integration of morphological data sets for phylogenetic analysis of Amniota: The importance of integumentary characters and increased taxonomic sampling: Systematic Biology, 54, 1-18.

Hill, R.V., 2006, Comparative anatomy and histology of xenarthrans osteoderms: Journal of Morphology, 267, 1441-1460.

Illiger, C., 1811, Prodromus systematis mammalium et avium: additis terminis zoogeographicis utriusque classis, eorumque versione germanica: Berlin, C. Salfeld.

Instituto Nacional de Estadística, Geografía e Informática (INEGI), 1992, Síntesis Geográfica del estado de Hidalgo: México, D. F., Instituto Nacional de Estadística, Geografía e Informática.

Kurtén, B., Anderson., E., 1980, Pleistocene mammals of North America: New York, Columbia University Press.

McDonald, H.G., 2002, Fossil Xenarthra of Mexico: a review, en Montellano-Ballesteros, M., Arroyo-Cabrales, J. (eds.), Avances en los estudios paleomastozoológicos: México, D. F., Instituto Nacional de Antropología e Historia, Serie Arqueología, 227-248.

Mead, J.I., Swift, S.L., White, R.S., McDonald, H.G, Báez, A., 2007, Late Pleistocene (Rancholabrean) Glyptodont and Pampathere (Xenarthra, Cingulata) from Sonora, México: Revista Mexicana de Ciencias Geológicas, 24, 439-449.

Montellano-Ballesteros, M., 1992, Una edad del Irvingtoniano al Rancholabreano para la fauna Cedazo del estado de Aguascalientes: Revista del Instituto de Geología, Universidad Nacional Autónoma de México, 9, 195-203.

Mooser, O., Dalquest, W.W., 1975, Pleistocene mammals from Aguascalientes, central Mexico: Journal of Mammalogy, 56, 781820 .

Osborn, H.F., 1903, Glyptotherium texanum, a new glyptodont, from the Lower Pleistocene of Texas: Bulletin of the American Museum of Natural History, 19, 491-494.

Roth, J.K.A., Laerm., J., 1980, A late Pleistocene vertebrate assemblage from Edisto Island, South Carolina: Brimleyana, 3, 1-29.

Silva-Bárcenas, A., 1969, Localidades de vertebrados fósiles en la República Mexicana: Universidad Nacional Autónoma de México, Instituto de Geología, Paleontología Mexicana, 28, 1-34.

Simpson, G.G., 1929, Pleistocene mammals fauna of the Seminole Field, Pinellas County, Florida: Bulletin of the American Museum of Natural History, 56, 561-599.

Simpson, G.G., 1980, Splendid isolation, The curious history of South American mammals: New Haven, United States of America, Yale University Press. 
Webb, S.D., 2001, Mammalia 2 Xenarthrans, in Hulbert Jr., R.C. (ed.), The fossil vertebrates of Florida: United States of America, University Press of Florida, 175-187.

Webb, S.D., Rancy, A., 1996, Late Cenozoic evolution of the Neotropical mammal fauna, in Jackson, J.B.C., Budd, A.F., Coates, A.G. (eds.), Evolution and Environment in Tropical America: United States of America, Chicago University Press, 335-358.
Manuscrito recibido: Febrero 27, 2009.

Manuscrito corregido recibido: Junio 1, 2009.

Manuscrito aceptado: Junio 5, 2009. 